\title{
Analyse optimaler Bestellmengen für unterschiedliche Preisabsatzfunktionen und Rabattarten
}

\author{
Matthias Forster*, Victoria Scholl
}

\section{Zusammenfassung}

Die Beschaffung von Gütern umfasst die Bereitstellung des Materials in der optimalen Menge. EOQ-Modelle (Economic Order Quantity) stellen eine Hilfe zur Optimierung dieser dar. Frühere Studien beschäftigten sich bereits mit Verfahren zur Berechnung der optimalen Bestellmenge bei einer preisabhängigen Nachfrage und mengenabhängigen Preisen. Burwell et al. entwickelten ein Verfahren zur Bestellmengenoptimierung mit preisabhängiger Nachfrage und Mengenrabatten für den Einkaufspreis und die Frachtkosten. Der Mengenrabatt ist entweder inkrementell oder ein Gesamtmengenrabatt. Burwell et al. betrachten die Nachfrage für den Monopolfall. Wir untersuchen den Konkurrenzfall. Die angegebene Beispielrechnung veranschaulicht die Methode.

\begin{abstract}
The procurement of commodities covers supply of the optimal quantities of material. EOQ models are a means to compute optimal quantities. Previous studies have dealt with algorithms that find optimal lot sizes when demand depends on price and quantity discounts are offered. Burwell et al. developed an algorithm to optimize order quantities when demand is considered to be dependent upon price and incorporate quantity and freight discounts. The quantity discount is either incremental or all-unit. Burwell et al. consider the monopolistic case. We study the case of competition. A numerical example is given that illustrates the method.
\end{abstract}

\section{Einleitung}

Jeder Produktionsbetrieb benötigt Material. Das Material kann der Betrieb entweder (a) verbrauchssynchron beschaffen oder (b) eine Einzelbeschaffung im Bedarfsfall vornehmen oder (c) ein Materiallager nutzen. Das Prinzip (a) verwendet kein Lager und verursacht daher keine Kapitalkosten. Jede Unterbrechung des Materialflusses führt sofort zur Unterbrechung der Produktion, wenn z. B. Liefertermine nicht eingehalten werden. In diesem Falle können enorme Kosten entstehen. Das Prinzip (b) setzt auf Einzelentscheidungen, die sich nicht verallgemeinern lassen. Die Lagerung (c) setzt eine Bestellpolitik voraus, d.h. eine allgemeine Regel. Bestellpolitiken lassen sich mit wissenschaftlichen Methoden untersuchen. Frühe Analysen zur optimalen Lagerverwaltung gehen auf Harris (im deutschsprachigen Raum auf Andler) zurück (Economic Order Quantity, kurz: EOQ-Formel).
Das Harris-Modell ist weit entfernt von der Wirklichkeit. Es wurde daher von vielen Autoren in verschiedenen Aspekten um mehrfache Erweiterungen ergänzt. Oft gehören zu den Erweiterungsaspekten neben Bestellrückständen, Kapazitätsbeschränkungen und Rabatten auch verschiedene Arten des Nachfrageverlaufs. Im Modell von Burwell et al. (1997), welches durch Chang (2013) wegen eines Fehlers verbessert wurde, werden die lineare und die multiplikative Preisabsatzfunktion im Monopol betrachtet. In der Masterarbeit „Bestellmengenoptimierung mit verschiedenen Preisabsatzfunktionen unter der Berücksichtigung von Mengen- und Frachtrabatten" wurde das Burwell-Chang-Modell um Preisabsatzfunktionen erweitert, die charakteristisch sind für Marktformen, in denen Konkurrenz vorhanden ist (Scholl 2014). Zu den verwendeten Preisabsatzfunktionen gehören die multiplikative unter der Bedingung von Konkurrenz sowie die einfach- und doppeltgeknickte. Diese Modelle finden in mehreren Marktformen Anwendung und können somit in vielen Unternehmen angewendet werden.

\section{Algorithmus}

Der Algorithmus von Chang (2013) und die Arbeit von Burwell et al. (1997) dienen als Grundlage für die Betrachtung von weiteren Preisabsatzfunktionen. Chang verbesserte den nicht ganz ausgereiften Algorithmus von Burwell et al. im Fall von gemischten Rabatten.

Der Algorithmus dient der Lösung des Problems der Mengenrabatte, dabei werden zwei Rabattarten verwendet, die den Einkaufspreis und die Frachtkosten umfassen. Der Algorithmus maximiert den Gewinn. Dabei ist die Nachfrage nach dem Gut preisabhängig und wird hier durch zwei Preisabsatzfunktionen dargestellt. Diese sind die lineare und die multiplikative Preisabsatzfunktion für den Monopolfall. Die Rabattformen sind erstens der Ge- 
samtmengenrabatt (all-unit discount) und der inkrementelle Rabatt, die jeweils sowohl auf den Einkaufspreis als auch auf die Kosten der Lieferung angewendet werden. Dadurch entstehen vier verschiedene Rabattmöglichkeiten:

a) all-unit Rabattstruktur für den Einkaufspreis und die Frachtkosten

b) inkrementelle Rabattstruktur für den Einkaufspreis und die Frachtkosten

c) all-unit Rabatt für den Einkaufspreis und inkrementeller Rabatt für die Frachtkosten

d) inkrementeller Rabatt für den Einkaufspreis und all-unit Rabatt für die Frachtkosten

Damit der Algorithmus den maximalen Gewinn bestimmen kann, müssen folgende Prämissen erfüllt sein:

a) Die Nachfrage ist gegeben durch eine Nachfragefunktion und diese ist verkaufspreisabhängig.

b) Die Dauer der Bestellung plus Lieferung ist bekannt und konstant.

c) Es wird ein Produkt betrachtet.

d) Es existieren keine Kapazitätsbeschränkungen.

e) Der Planungszeitraum ist unendlich.

f) Der Einkaufspreis ist bekannt und mengenabhängig.

g) Die Frachtkosten sind bekannt und gewichtsabhängig.

h) Das Gewicht der Ware ist bekannt und konstant.

i) Die restlichen Beschaffungskosten sind bekannt und konstant.

j) Fehlmengen, Produktfehler, verminderte Produktqualität und Nachlieferungen sind nicht erlaubt.

k) Die Lagerhaltungskosten sind bekannt und konstant.

I) Wertverluste und Entsorgungskosten sind nicht erlaubt.

m)Die variablen Marketingkosten sind bekannt und konstant.

Sei $Q$ die Bestellmenge und $v$ der Einkaufspreis. Dann stellt Tab. 1 die Rabattstruktur für den Preis des Produktes dar (Burwell et al. 1997: $143 \mathrm{ff}$.), d. h. die Bestellmenge $Q$ wird in $n+1$ Intervalle eingeteilt. Ist $Q$ in Intervall $i$, dann ist der Einkaufspreis $v_{i}$. $W$ sei das Gewicht der Bestellung. Dann stellt Tab. 2 die Rabattstruktur für den Preis der Lieferung dar, d.h. das Gewicht W wird in $l+l$ Intervalle eingeteilt. Ist $W$ in Intervall $i$, dann ist der Einkaufspreis $Y_{i}$.

Tab.1: Die Rabattstaffel für den Produktpreis

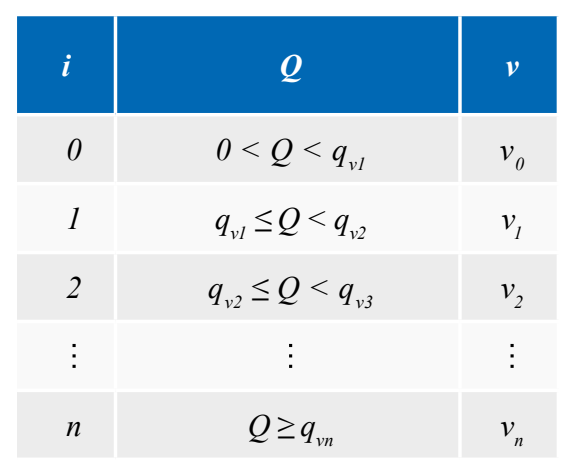

Tab.2: Die Rabattstaffel für Frachtkosten

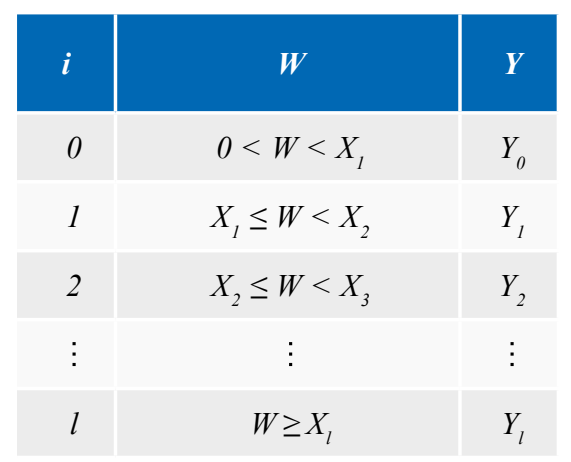

Der Produktpreis ist mengenabhängig. Die Frachtkosten sind gewichtsabhängig. Aus den beiden einzelnen Rabattstaffeln kann durch Kombination ein Rabattplan aufgebaut werden mit der Bedingung $f \leq l+n$ (Tab. 3).

Tab.3: Kombinierte Rabattstruktur

\begin{tabular}{c|c|c|}
\hline $\boldsymbol{i}$ & $\boldsymbol{Q}$ & $\boldsymbol{s}$ \\
\hline 0 & $0<Q<q_{s 1}$ & $s_{0}$ \\
\hline 1 & $q_{s 1} \leq Q<q_{s 2}$ & $s_{1}$ \\
\hline 2 & $q_{s 2} \leq Q<q_{s 3}$ & $s_{2}$ \\
\hline$\vdots$ & $\vdots$ & $\vdots$ \\
\hline$f$ & $Q \geq q_{s f}$ & $s_{f}$ \\
\hline
\end{tabular}

Im inkrementellen Rabattfall gilt der Rabatt ausschließlich für die Mengen im Intervall, während im Gesamtmengenrabattfall (all-unit discount) der Rabatt für alle gekauften Produkte gilt. Um dieser Tatsache Rechnung zu tragen, sind zusätzliche fixe Kos- tenanteile für inkrementelle Rabatte nötig.

\subsection{Modellbeschreibung}

Es gibt Modelle für unterschiedliche Kombinationen von Rabatten auf den Einkaufspreis bzw. auf die Frachtkosten. Allen Modellen gemeinsam ist (a) das Ziel der Gewinnmaximierung, (b) die Bestimmung des optimalen Verkaufspreises $p$ und der optimalen Bestellmenge $Q$ und (c) die Berücksichtigung der Nachfragefunktion $D=D(p)$. Wir betrachten in diesem Zusammenhang zum ersten Mal Nachfragefunktionen, die sich durch ihre Praxisrelevanz auszeichnen. Sie wurden in der Fachliteratur bisher nicht berücksichtigt. Die Kombinationen bestehen aus (a) inkrementellem Rabatt auf den Einkaufspreis und die Frachtkosten, (b) all-unit Rabatt auf den Einkaufspreis und die Frachtkosten und (c) gemischtem inkrementellem bzw. all-unit Rabatt auf den Einkaufspreis und die Frachtkosten (Burwell et al. 1997).

Grundsätzliche Annahmen, die für alle Rabattfälle gelten: Es wird der Gewinn optimiert, mit den Variablen Verkaufspreis $p$ und Menge $Q$. Die Nachfrage ist eine Funktion des Preises $p$ mit $D=D(p)$.

Folgende Parameter werden für das Grundmodell (Burwell et al. 1997) verwendet:

$\Pi$ Gewinnfunktion

$i, j$ Indexe für die Rabattstaffeln

$C_{o}$ Bestellkosten

$C_{m} \quad$ variable Marketingkosten

$v_{j} \quad$ mengenabhängiger Einkaufspreis

$g$ stückbezogene Frachtkosten $(g=w Y)$

$s \quad$ kombinierte Stückkosten $(s=v+g)$

$H$ notwendig für die Beschaffungskosten im inkrementellen Rabattfall

$Z$ notwendig für die Frachtkosten im inkrementellen Rabattfall

$t \quad$ Lieferzeit für eine Bestellung

$r \quad$ Lagerhaltungskosten innerhalb der Lieferung

$R \quad$ Lagerhaltungskosten nach der Lieferung

$q_{s i} \quad$ Mengenrabattschwelle für den Einkaufspreis

$q_{v i}$ Mengenrabattschwelle für die kombinierten Einkaufspreise

$W \quad$ Liefergewicht für eine Bestellung mit der Bestellmenge $Q$

$w \quad$ Liefergewicht pro Stück 
$Y$ gewichtsabhängiger Frachtkostenanteil pro Stück

$X_{i}$ Gewichtsrabattschwelle für das Gewicht pro Stück für die Frachtkosten $Y_{i}$

$\lambda$ Konvergenzparameter

Für den Fall von fixen Einkaufskosten

$s=v+g$ ist der Gewinn für den Käufer:

$$
\begin{aligned}
& \text { (1) } \Pi(p, Q)=\left\{p-s-v r t-C_{m}-\frac{\left[H(1+r t)+Z+C_{0}\right]}{Q}\right\} D-(H+Z+s Q) \frac{R}{2} \text {. } \\
& \text { (2) } H=\sum_{b=1}^{i}\left(q_{s_{b}}-1\right)\left(v_{b-1}-v_{b}\right) \\
& \text { (3) } Z=\sum_{b=1}^{i}\left(q_{s_{b}}-1\right)\left(q_{b-1}-q_{b}\right) .
\end{aligned}
$$

Das ist so zu verstehen: Für $s=s_{i}$ sind $H$ und $Z$ zusätzliche Fixkosten auf den Einkaufspreis und die Frachtkosten für den inkrementellen Rabattfall, um diesen richtig darzustellen (Burwell et al. 1997).

Die Ableitung nach $p$ und $Q$ :

$$
\begin{aligned}
& \text { (4) } \frac{\partial \Pi}{\partial p}=D+\left(p-s-v r t-C_{m}-\frac{H(1+r t)+Z+C_{0}}{Q}\right) D^{\prime} \text {. } \\
& \text { (5) } \frac{\partial \Pi}{\partial Q}=\left(H(1+r t)+Z+C_{0}\right) \frac{D}{Q^{2}}-\frac{s R}{2} \text {. }
\end{aligned}
$$

Die zweite Ableitung nach $p$ :

$6 \frac{\partial^{2} \Pi}{\partial p^{2}}=2 D^{\prime}+\left(p-s-v r t-C_{m}-\frac{H(1+r t)+Z+C_{0}}{Q}\right) D^{\prime \prime}<0$.

Die Annahme (6) garantiert, dass $\Pi$ eine konkave Funktion von $p$ ist, wenn fix ist. Der optimale Wert für $p$ mit einem fixen Wert für $Q$ lässt sich durch $p_{\text {opt }}(Q)$ die (= durch die Gleichung) (4) bestimmen. Die Annahme (6) zeigt, dass der optimale Wert $p_{\text {opt }}(Q)$ eine reellwertige Funktion von $Q$ für die lineare und multiplikative Preisabsatzfunktion ist. Burwell et al. (1997) substituieren die Funktion in Gleichung (1):

(7) $\Pi_{1}(Q)=\Pi\left[p_{\text {opt }}(Q), Q\right]$.

Abad (1988) zeigt, dass $\Pi_{l}(Q)$ eine konvex-konkave Funktion von $Q$ ist. Eine konvex-konkave Funktion wie $\Pi_{l}(Q)$ hat ihr Optimum entweder im Punkt $Q=0$ oder $Q=Q_{o p t}$, wenn $Q_{\text {opt }}$ $>0$. Wenn das Optimum bei $Q=0$ ist, ist es nicht profitabel, das Produkt zu kaufen. Weiter nutzen Burwell et al. das iterative Verfahren von Abad, um $Q_{\text {opt }}$ zu finden. So kann $Q_{\text {opt }}$ und der dazugehörige Preis $p_{\text {opt }}\left(Q_{o p t}\right)$ für ein fixes $s$ gefunden werden. Das iterati- ve Verfahren, erstmals beschrieben in Abad (1988), verwenden Burwell et al. ebenfalls:

Schritt 1:

$k=0$ und $Q^{0}=\infty$

Schritt 2:

Berechne $p_{\text {opt }}\left(Q^{k}\right)$, nutze dafür $\frac{\partial \Pi}{\partial p}$

Schritt 3:

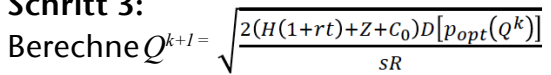

Schritt 4:

Wenn $\left|Q^{k+1}-Q^{k}\right| \geq \lambda$, dann $k=k+1$ und gehe zu Schritt 2 .

Im Fall (a) von inkrementellem Produktpreis und inkrementellen Frachtkosten sieht der Algorithmus wie folgt aus:

\section{Schritt 1:}

Berechne $Q_{\text {opt }}$ und $p_{\text {opt }}$ für jedes $s_{i}$ wie oben beschrieben.

Schritt 2:

Berechne den Gewinn $\Pi\left(p_{o p t} Q_{o p}\right)$ für jeden gültigen Wert $Q_{o p t} ; Q_{o p t}$ ist gültig, wenn $q_{s i} \leq Q_{o p t}<q_{s_{i+1}}$ und $s=s_{i}$.

\section{Schritt 3:}

Die optimale Losgröße und der optimale Preis sind die gültigen $Q_{\text {opt }}$ und $p_{\text {opt }}$, die zu dem größten positiven Gewinn aus Schritt 2 gehören. Wenn kein positiver Gewinn gefunden werden konnte, dann ist die beste Lösung $Q=0$ (Burwell et al. 1997).

Die zusätzlichen Fixkosten $H$ bzw. $Z$ auf den Einkaufspreis und die Frachtkosten sind im Falle des inkrementellen Rabattes ungleich 0 und werden deshalb nach den Gleichungen (2) und (3) berechnet.

Im Fall (b) von all-unit Rabatt sowohl auf den Einkaufspreis als auch auf die Frachtkosten sieht der Algorithmus wie folgt aus:

\section{Schritt 1:}

Für jede Kostenkombination $s=s_{i}$ berechne $Q_{i_{\text {opt }}}$ unter der Annahme eines fixen $s$. Identifiziere den größten Indexwert $I$, bei dem $q_{s i} \leq Q_{o p t}<q_{s_{i+1}}$ ist.

\section{Schritt 2:}

Berechne $\Pi\left(p_{o p t} Q_{o p}\right)$ für $s=s_{i}$.

\section{Schritt 3:}

Für jedes $j>i$ berechne $\Pi\left(p_{o p t}\left(q_{s j}\right), Q_{o p}\right)$ mit $s=s_{j}$.

\section{Schritt 4:}

Vergleiche die Gewinnlevel aus Schritt 2 und 3. Wähle das $Q$ aus, welches den größten Gewinn erzeugt. Falls dieser negativ sein sollte, kaufe das Produkt nicht.

Die zusätzlichen Fixkosten $H$ bzw. $Z$ auf den Einkaufspreis und die Frachtkosten sind im Falle des all-unit Rabattes beide gleich 0 (Burwell et al. 1997).

Im Fall (c) des gemischten inkrementellen und all-unit Rabattes auf den Einkaufspreis und die Frachtkosten sieht der Algorithmus wie folgt aus:

\section{Schritt 1:}

Berechne $p_{o p t}, Q_{o p t}$ und $\Pi\left(p_{o p t}, Q_{o p}\right)$ für jeden Index $I$ in der kombinierten Rabattstruktur.

\section{Schritt 2:}

Sortiere die Ergebnisse aus Schritt 1 in gültige und ungültige Ergebnisse. 


\section{Schritt 3:}

Finde den größten $\Pi\left(p_{o p t}, Q_{o p}\right)$ aus den gültigen Ergebnissen und betitle inn mit $\Pi^{L}\left(p_{o p t}, Q_{o p}\right)$.

\section{Schritt 4:}

Die ungültigen Ergebnisse aus den Indexen $j$ werden mit $\Pi^{L}\left(p_{o p p} Q_{o p}\right)$ verglichen. Ist $\Pi\left(p_{o p t} Q_{o p p}\right)>\Pi^{L}\left(p_{o p t} Q_{o p p}\right)$, dann nimm $q_{s_{j}}$ und berechne $p_{\text {opt }}\left(q_{s_{j}}\right)$ und $\Pi\left(q_{s j}, p_{\text {opt }}\left(q_{s j}\right)\right)$, sonst ignoriere $q_{s j}($ Chang 2013).

\section{Schritt 5:}

Vergleiche $\Pi^{L}\left(p_{\text {opt }} Q_{o p}\right)$ mit allen $\Pi\left(q_{s_{j}}, p_{\text {opt }}\left(q_{s_{j}}\right)\right)$ aus Schritt 4 gefundenen. Wähle das $Q$ mit dem größten Gewinn.

Im Fall des gemischten Rabattes ist $H=0$ oder $Z=0$. Das bedeutet, wenn der Einkaufspreis all-unit Rabatt $H=0$ beinhaltet, beinhalten die Frachtkosten inkrementellen Rabatt. $Z$ wird bestimmt durch (3).

\subsection{Modifikation durch die lineare Nachfragefunktion}

Das verwendete lineare Modell mit Konkurrenz sieht wie folgt aus:

$$
\text { (8) } D(p)=a-b p+c \bar{p} \quad a, b, c>0, p \in\left(0, \frac{a+c \bar{p}}{b}\right) \text {. }
$$

Der Parameter $a$ stellt die Sättigungsmenge, $b$ den Anstieg $\bar{p}$ den Konkurrenzpreis und $c$ die Wirkung des Konkurrenzpreises dar. Die Gewinnfunktion für den Fall von fixer Kostenkombination $s$ und der linearen Preisabsatzfunktion ist im Folgenden dargestellt, dabei ist immer vorausgesetzt $p, Q>0$ :

$$
\text { (9) } \Pi_{i}(Q, p)=\left\{p-s-v r t-C_{m}-\frac{\left[H(1+r t)+Z+C_{0}\right]}{Q}\right\}(a-b p+c \bar{p})-(H+Z+s Q) \frac{R}{2} \text {. }
$$

Vorausgesetzt (Scholl 2014: $60 \mathrm{ff}.): a, b,\left(H(1+r t)+Z+C_{0}\right)>0$ und $a+c \bar{p}>b\left(s+v r t+c_{m}\right)$. Dabei verändern sich die zusätzlichen fixen Kosten für den inkrementellen Rabatt nicht. Aus Gleichung (4) wird

$$
10 p=\frac{a+c \bar{p}}{2 b}+\frac{1}{2}\left(s+v r t+C_{m}+\frac{\left(H(1+r t)+Z+C_{0}\right)}{Q}\right) \text {. }
$$

Der optimale Preis ist $p_{\text {opt }}$ Annahme (10) zeigt eine reelle Funktion in Abhängigkeit von $Q$ und mit $p=p_{\text {opt }}(Q)$. Wird nun $p_{\text {opt }}(Q)$ in Gleichung (1) substituiert, ergibt sich $\Pi_{l}\left(p_{\text {opt }}(Q), Q\right)$ (Burwell et al. 1997, Abad 1988). Für das lineare Modell mit Konkurrenz heißt das

(11) $\Pi_{1}(Q)=\frac{b}{4}\left(\frac{a+c \bar{p}}{b}-s-v r t-C_{m}-\frac{\left(H(1+r t)+Z+C_{0}\right)}{Q}\right)^{2}-(H+Z+s Q) \frac{R}{2}$.

Aus Gleichung $\frac{d \Pi_{1}(Q)}{d Q}=0$ wird im linearen Fall mit Konkurrenz

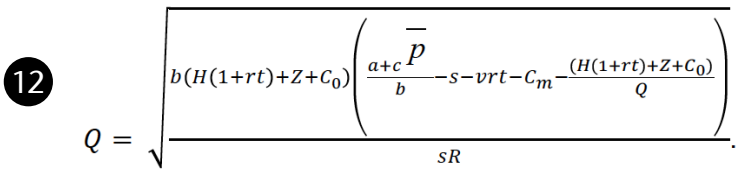

$Q$ ist eindeutig wenn $c \bar{p}+a \neq b\left(s+v r t+C_{m}\right)$, die Beweisführung zeigt aber auch, dass $c \bar{p}+a>b\left(s+v r t+C_{m}\right)$ und somit $Q$ immer eindeutig ist.

\subsection{Modifikation durch die multiplikative Nachfragefunktion}

Das verwendete multiplikative Modell mit Konkurrenz sieht wie folgt aus:

$$
13 \quad D(p)=a\left(\frac{p}{\bar{p}}\right)^{b} \quad \text { mit } a, b, p, \bar{p}>0, b \neq 1 \text { und } p<\frac{a}{b} \text {. }
$$

Der Parameter $b$ gibt die Preiselastizität an und $a$ stellt die minimale Absatzmenge dar. Gleichung (14) ist die Gewinnfunktion für den Fall von fixer Kostenkombination $s$ und der multiplikativen Preisabsatzfunktion, dabei ist immer vorausgesetzt $p, Q>0$ :

$14 \Pi_{i}(Q, p)=\left\{p-s-v r t-C_{m}-\frac{\left[H(1+r t)+Z+C_{0}\right]}{Q}\right\} a\left(\frac{p}{\bar{p}}\right)^{b}-(H+Z+s Q) \frac{R}{2}$. 
Vorausgesetzt $\left(H(1+r t)+Z+C_{0}\right),\left(s+v r t+C_{n}\right)>0, b>2$ ist erfüllt (Scholl 2014: 60 ff.). Dabei verändern sich die zusätzlichen fixen Kosten für den inkrementellen Rabatt nicht. Aus Gleichung (4) wird

$15 p=\frac{b}{b-1}\left(s+v r t+C_{m}+\frac{\left(H(1+r t)+Z+C_{0}\right)}{Q}\right)$.

Der optimale Preis ist $p_{\text {opt }}$. Annahme (15) zeigt eine reelle Funktion in Abhängigkeit von $Q$ und mit $p=p_{\text {opt }}(Q)$. Wird nun $p_{\text {opt }}(Q)$ in Gleichung (1) substituiert, dann ergibt sich $\Pi_{I}\left(p_{\text {opt }}(Q), Q\right)$. Für das multiplikative Modell mit Konkurrenz heißt das

๑ $\Pi_{1}(Q)=\frac{a}{b\left(\bar{p}^{b}\right)}\left(s+v r t+C_{m}+\frac{\left(H(1+r t)+Z+C_{0}\right)}{Q}\left(\frac{b}{b-1}\right)\right)^{v+1}-(h+Z+s Q) \frac{R}{2}$

Aus Gleichung $\frac{d \Pi_{1}(Q)}{d Q}=0$ wird im multiplikativen Fall mit Konkurrenz

(17) $Q=\sqrt{\frac{2\left(H(1+r t)+Z+C_{0}\right)}{s R} \frac{a}{\bar{p}^{b}}\left(\frac{b}{b-1}\left(s+v r t+C_{m}+\frac{\left(H(1+r t)+Z+C_{0}\right.}{Q}\right)\right)^{b}}$.

Tab. 4: Variablen mit mengenabhängigen Preisen

\begin{tabular}{|c|c|c|c|c|c|c|}
\hline $\boldsymbol{l}$ & $\boldsymbol{Q}$ & $\boldsymbol{V}$ & $\boldsymbol{V}$ & $g$ & $\boldsymbol{H}$ & $\boldsymbol{Z}$ \\
\hline 0 & $1<Q<500$ & 5,50 & 4,50 & 1,00 & 0,00 & 0,00 \\
\hline 1 & $500 \leq Q<1.000$ & 5,30 & 4,50 & 0,80 & 0,00 & 99,80 \\
\hline 2 & $1.000 \leq Q<1.250$ & 4,80 & 4,00 & 0,80 & 0,00 & 99,80 \\
\hline 3 & $1.250 \leq Q<10.000$ & 4,60 & 4,00 & 0,60 & 0,00 & 349,60 \\
\hline 4 & $10.000 \leq Q$ & 4,30 & 3,70 & 0,60 & 0,00 & 349,60 \\
\hline
\end{tabular}

Tab. 5: Werte für die Nachfragefunktionen

\section{Werte für die lineare Funktion:}

$\bar{p}=9,50$

$c=589,47$

$b=900$

$a=6.800,00$

Tab. 6: Die optimalen Gewinne im Überblick

\begin{tabular}{|l|c|c|c|c|}
\hline Nachfragefunktion & Gewinn $\boldsymbol{\Pi}$ & Preis $\boldsymbol{p}$ & Menge $\boldsymbol{Q}$ & Index \\
\hline Multiplikativ & $16.237,30$ & 8,8738 & 1.999 & 3 \\
\hline Linear & $12.106,09$ & 9,8955 & 1.509 & 1 \\
\hline
\end{tabular}

\section{Fallbeispiele}

Das Fallbeispiel zeigt den Einsatz des Algorithmus mit Verwendung der linearen und multiplikativen Preisabsatzfunktion mit Konkurrenz. Die Berechnungen wurden mit dem Programm MATLAB R2013a mit der Version: 8.1.0.604 (R2013a) durchgeführt.

Das Beispiel ist in Anlehnung an Chang (2013) gewählt worden und stellt einen gemischten Rabattfall dar.

Die Tab. 4 zeigt die Preise für die variablen mengenabhängigen Parameter $\mathrm{s}, \mathrm{v}, \mathrm{g}, \mathrm{H}$ und $\mathrm{Z}$ für das Beispiel. Im Beispiel sind somit 5 verschiedene Mengenoptionen enthalten, in den Indexen I 0-4.

Die konstanten Parameter für das Beispiel sind:

$$
\begin{aligned}
R & =0,40 \\
r & =0,20 \\
t & =0,02 \\
C_{0} & =250,00 \\
C_{m} & =1,00
\end{aligned}
$$

Die Werte für die einzelnen Nachfragefunktionen sind gesondert in Tab. 5 dargestellt. Sie sind frei gewählt.

In Tab. 6 findet man die optimalen Gewinne für die beiden Nachfragefunktionen. Abb. 1 zeigt den Gewinn in Abhängigkeit von der Bestellmenge für beide Nachfragefunktionen.

\section{Praxisbezug}

Der Algorithmus aus Kapitel 2 berücksichtigt verschiedene Kosten: Bestellkosten, variable Marketingkosten, mengenabhängiger Beschaffungspreis, Lagerhaltungskosten innerhalb der Lieferzeit und danach und gewichtsabhängige Frachtkosten. Weiter sind 4 Kombinationen von Rabattarten enthalten, der Gesamtmengenrabatt (all-unit) und der inkrementelle Rabatt, welche jeweils auf die Frachtkosten und den Beschaffungspreis angewendet werden können. Mit Hilfe des Algorithmus wird der Gewinn optimiert und der Verkaufspreis berechnet. Der Gewinn wird mithilfe einer preisabhängigen Nachfrage optimiert; dazu wurden vier Varianten von Nachfragefunktionen genutzt: die lineare und multiplikative Preisabsatzfunktion, jeweils mit und ohne Konkurrenz. Der beschriebene Algorithmus geht 


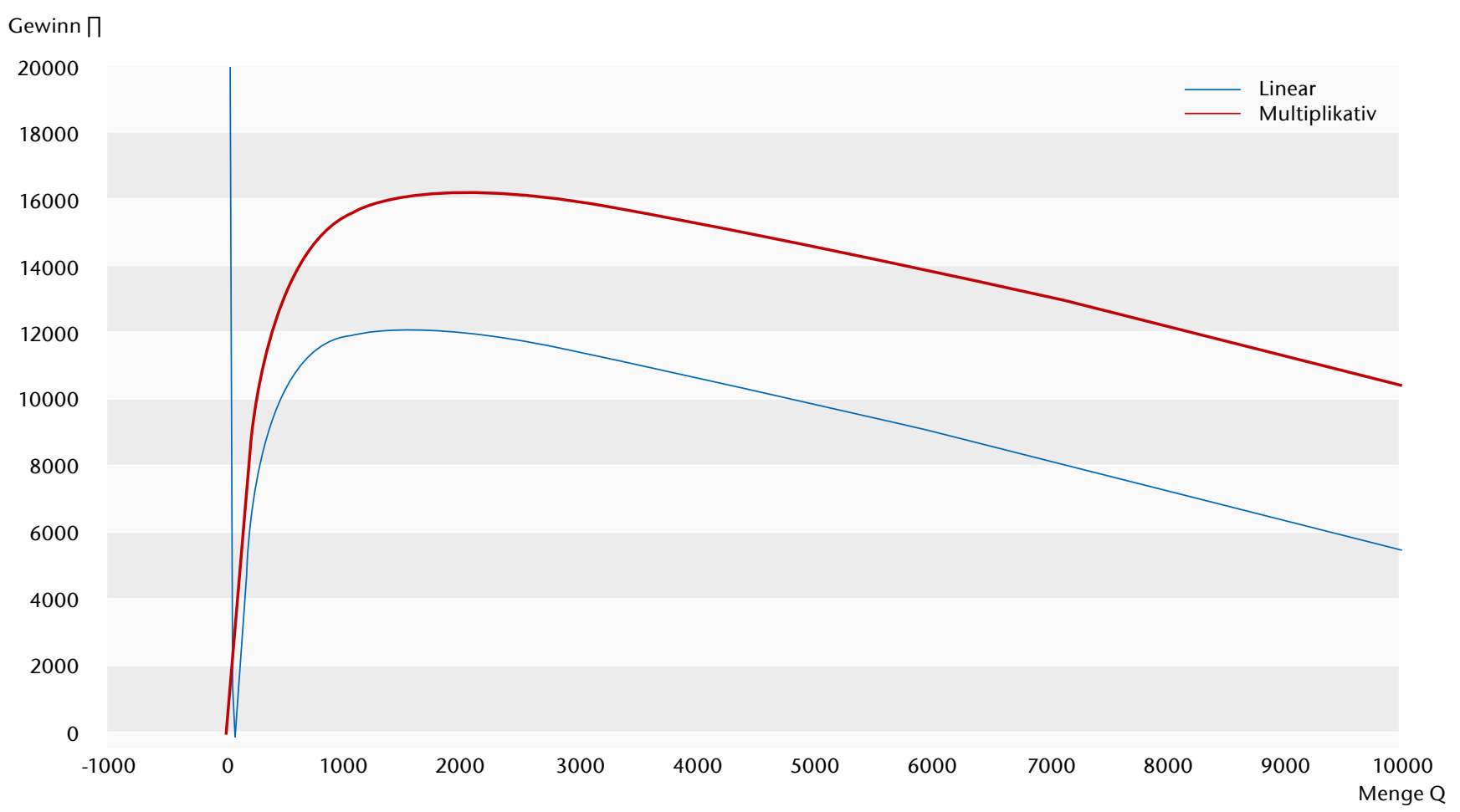

Abb. 1) Gewinn in Abhängigkeit von der Bestellmenge

von Voraussetzungen aus, die in der Praxis nicht immer erfüllt sind. Dazu zählen: Es wird ausschließlich ein Produkt betrachtet. Es handelt sich um ein statisches Modell, d. h., die Kosten ändern sich im Zeitablauf nicht. Das Lager kann jede Liefermenge aufnehmen (theoretisch unendliche Lagerkapazität). Fehlmengen, Produktfehler, verminderte Produktqualität, Schwund, Entsorgungskosten und Nachlieferungen werden im Algorithmus nicht berücksichtigt. Der Verkaufspreis wird mit Hilfe des Algorithmus berechnet. Produktionskosten werden nicht beachtet, sodass eine Anwendung nur für Güter ohne Weiterverarbeitung sinnvoll erscheint. Der Algorithmus bestimmt den Verkaufspreis und die Rabatte auf den Einkaufspreis und auf die Frachtkosten. Produkte, die einer gesetzlichen Preisbindung unterliegen oder bei denen mögliche Rabatte eingeschränkt sind, sind ungeeignet. Einzelne Güter unterliegen der gesetzlichen Preisbindung in Deutschland. Hierzu zählen unter anderem verlagsund buchhandelstypische Produkte und Tabakwaren ( $\S 2$ BuchPrG Abs. 1 Nr. 3 und 4; § 26 TabStG Abs. 1). Durch den Wegfall des Rabattgesetzes sind Unternehmen weitestgehend frei in der Vergabe von Rabatten ( $\S 1$ GWB Abs. 2). Der Algorithmus ist für die Verwendung im Bereich der Distribution gedacht (Großhandel und Einzelhandel).

Verschiedene Studien beschreiben den Bedarf von Handelsunternehmen: Die Studie von Fauser et al. (1995), in der 81 Unternehmungen befragt wurden, beschreibt das Handelslager vor allem im Hinblick auf die Bestellmengen und Bestellzeitpunkte. Die Studie zeigt, dass $81 \%$ der befragten Unternehmungen eine EDV-gestützte Lagerführung verwenden. Die Hälfte der befragten Händler führen zwischen 5.000 und 50.000 Güter. Bei $50 \%$ der Befragten ist die Nachfrage deterministisch, also im Voraus bekannt, und etwa $38 \%$ davon besitzen gleichzeitig eine relativ gleichbleibende Nachfrage. Des Weiteren gaben die Unternehmungen an, nur geringe Nachfrageausfälle durch Lieferschwierigkeiten zu haben, aber eben auch keine genaue Erfassung durchzuführen. Sammelbestellungen nutzen $40 \%$ der Händler, eine Kombination aus Sammel- und Einzelbestellungen ist bei $28 \%$ zu beobachten und Einzelbestellungen werden hingegen von $32 \%$ der Händler verwendet. Weiter ist die Lagerkapazität als ein sehr wichtiger Aspekt beschrieben worden. Die Untersuchung zeigt: Händler verwenden vorwiegend statische Bestellmengenmodelle in der
Praxis. Fauser et al. empfehlen Handelsunternehmen mit einer großen Anzahl an Produkten, nur für einen Teil der Produkte aufwendige Verfahren zu nutzen.

Also können etwa $19 \%$ der befragten Unternehmen den vorgestellten Algorithmus anwenden, weil die Voraussetzung einer bekannten und gleichbleibenden Nachfrage gegeben ist. Für die einzelnen Produkte im Sortiment kann man davon ausgehen, dass die Lagerkapazität ausreicht, um die Bestellmenge zu lagern.

In Zusammenhang mit dem dargestellten Algorithmus wurden in der Fachliteratur bisher zwei Preisabsatzfunktionen für monopolistische Märkte untersucht. Wir haben in Kapitel 2.2 und 2.3 die Darstellung um zwei Nachfragefunktionen erweitert. Die Erweiterung bezieht das Polypol und das Oligopol auf unvollkommenen Märkten ein. Es handelt sich um häufige Marktformen und einen großen Einsatzbereich (Ott 1997: 170).

Eine weitere Studie aus dem Lebensmittelbereich beschreibt eine Befragung von 23 Herstellern zu deren Vertragsvereinbarungen mit ihren Kunden und zeigt, dass $75 \%$ der Händler und Hersteller einen Rahmenvertrag über die Dauer von einem Jahr abschließen (Schulze 1998: 257). In 
diesen Rahmenverträgen sind zu $95 \%$ bereits Preise und Rabatte und zu $90 \%$ Funktionsrabatte enthalten, dabei betrifft diese Studie der Funktionsrabatte unter anderem den logistischen Bereich (Schulze 1998: 65-66). Weiter sind wie oben beschrieben zumeist Mengen- und Lieferrabatte inbegriffen. Im beschriebenen Algorithmus sind also viele wichtige Aspekte aus Rahmenverträgen der betrieblichen Praxis enthalten.

\section{Zusammenfassende Schluss- betrachtung und Ausblick}

Die Nutzung eines Materiallagers setzt eine Bestellpolitik voraus, die unter gegebenen Bedingungen mit quantitativen Methoden untersucht werden kann. Der in dieser Arbeit vorgestellte Algorithmus dient der Optimierung von Bestellmengen. Der Algorithmus wurde von Burwell et al. entwickelt und von Chang korrigiert. Diese Untersuchung bezieht sich auf die lineare und multiplikative Preisabsatzfunktion im Konkurrenzfall. Durch unsere Modifikation des Algorithmus können Marktformen einbezogen werden, die zuvor nicht inbegriffen waren (Polypol und Oligopol). Das Modell wird so praxisnäher.

Der um weitere Preisabsatzfunktionen ergänzte Algorithmus wird beispielhaft im Kapitel Fallbeispiele vorgeführt. Die Beispiele zeigen, dass die untersuchte multiplikative und auch die lineare Funktion in den Algorithmus integriert werden können.

Die Diskussion der Anwendbarkeit in Kapitel 4 zeigt, dass die praktische Verwendung des Algorithmus möglich ist. Die Voraussetzungen sind aber in der Praxis nicht immer gegeben. Eine weitere Verbesserung könnte die Einführung von Kapazitätsrestriktionen sein. Des Weiteren werden viele Einflussfaktoren wie zum Beispiel die Produktqualität, markenpolitische Aspekte, Präferenzen und das Konkurrenzverhalten zu wenig in Preisabsatzfunktionen abgebildet.

\section{LITERATUR}

Abad PL (1988) Determining Optimal Selling Price and Lot Size When the Supplier Offers All-Unit Quantity Discounts. Decis Sci 19(3):622-634. doi: 10.1111/j.15405915.1988.tb00290.x

Burwell TH, Dave DS, Fitzpatrick KE, Roy MR (1997) Economic lot size model for price-dependent demand Economic lot size model for price-dependent demand 48(2):141-155. doi: 10.1016/S0925-5273(96)00085-0

Chang H (2013) A note on an economic lot size model for price-dependent demand under quantity and freight discounts. Int J Prod Econ 144(1):175-179. doi: 10.1016/j. ijpe.2013.02.001

Fauser M, Leeger T, Morlock M (1995) Einsatz von Lagerhaltungsmodellen bei Handelsunternehmen. OR Spektrum 17(1):31-35. doi: 10.1007/BF01719729

Ott AE (1997) Grundzüge der Preistheorie, 3rd edn. Grundriß der Sozialwissenschaft, vol 25. Vandenhoeck \& Ruprecht, Göttingen. ISBN: 978-3-525-10519-1

Scholl V (2014) Bestellmengenoptimierung mit verschiedenen Preisabsatzfunktionen unter der Berücksichtigung von Mengen- und Frachtrabatten. Masterarbeit, Technische Hochschule Wildau

Schulze M (1998) Nachfragemacht im Lebensmitteleinzelhandel. Ökonomische und kartellrechtliche Aspekte. Kasseler Wirtschafts- und Verwaltungswissenschaften, vol 8. Deutscher Universitätsverlag, Wiesbaden. ISBN 978-3-8244-6770-9. doi: 10.1007/978-3-322-95252-3

\section{AUTOREN}

Prof. Dr. rer. pol. Matthias Forster

Technische Hochschule Wildau

Betriebswirtschaftslehre mit dem Schwerpunkt Planung inner- und außerbetrieblicher DV-gestützter logistischer Prozesse

Victoria Scholl, M.A.

Ehemalige Studentin der Technischen Hochschule Wildau

E-Mail für Korrenspondenz:

matthias.forster@th-wildau.de 